\title{
Evaluation of Fast Generation Cycling in Oat (Avena sativa)
}

\author{
D.J. HeusChele ${ }^{1}$, A. $\mathrm{CASE}^{2}$ and K.P. SMith ${ }^{1 *}$ \\ ${ }^{1}$ Department of Agronomy and Plant Genetics, University of Minnesota, St. Paul, MN 55108 \\ ${ }^{2} \mathrm{AB}$ InBev - Global Barley Research, Fort Collins, CO 80524
}

(Received 8 May 2019;

Accepted 1 August 2019)

\begin{abstract}
Cereal breeding programs are interested in increasing the number of generations per year to reduce the time needed to develop new cultivars. A common method to accomplish this is to extend the photoperiod to speed up plant growth. For oat, this method is problematic because the species responds to changes in light and temperature. Current methods of fast generation cycling in oat require embryo rescue, which is labor intensive and has a low success rate. Recently a method was developed using increased photoperiod and foliar mineral supplement to reduce generation time for wheat and barley. We evaluated this newly published method in oat and found that anthesis occurred $15 \pm 3$ days faster, however there was a 3 -fold reduction in seed count and a 2-fold reduction in inflorescence weight. In addition, we measured endogenous ascorbate to evaluate the physiological status of the plants under fast generation cycling conditions. For oat, fast generation cycling would be effective to more rapidly advance populations using single seed descent, but not as useful when seed yield is important.
\end{abstract}

Keywords: fast generation cycling, oat, ascorbic acid

Abbreviations: AsA, reduced ascorbate; ROS, reactive oxygen stress

\section{Introduction}

Reducing the time from initial crossing to variety release is of interest to all plant breeders. In a typical spring cereal breeding program, breeders advance lines by bulking or single seed descent to the $\mathrm{F}_{4}-\mathrm{F}_{6}$ generation before conducting yield trials. Breeders attempt to increase the numbers of generations produced per year with the use of counterseason nurseries and glasshouses. While counter-season nurseries add one additional generation per year, two to three additional generations can be advanced per year with the use of a glasshouse (Forster et al. 2014). Unfortunately, in oat (Avena sativa) this technique has a $2-10 \%$ embryo rescue success rate of which only $20 \%$ survive to adulthood (Rines 2003). Even with better embryo rescue techniques (Liu et al. 2016), the process is still laborious for a breeding pipeline. Therefore, development of a reliable method to accelerate plant growth, and reduce generation time, with large numbers of segregating individuals within a breeding program is essential.

\footnotetext{
*Corresponding author; E-mail: smith376@umn.edu
} 
Recent work has shown that changing photoperiod and nutrient inputs can increase the number of generations per year to six for wheat (Triticum sp.) and barley (Hordeum vulgare; Watson et al. 2018) without embryo rescue. They suggested that the stress of a longer photoperiod accelerated developmental rates in this fast generation technique. In oat, a method of fast generation cycling has been developed by increasing photoperiod (Liu et al. 2016), however, this technique still requires embryo rescue. Oat is more environmentally sensitive than other cereals to certain abiotic stresses including light (Quiles 2006). Therefore, growing oat in growth chambers or glasshouses at an accelerated rate with fast generation methods suggested by Watson et al. (2018) could be problematic.

Physiological changes, such as growth rate, have been reported to occur in response to abiotic stresses, such as light, temperature and root growth space. An increase in growth rate for wheat and barley occurs with above optimal temperatures (Chowdhury and Wardlaw 1978) and light radiation (Stockman et al. 1983; Fischer 1985). However, in the case of oat, growth eventually plateaus as temperature and light radiation increase due to the inhibition photosynthesis (Quiles 2006).

Environmental signals, such as light duration, act as triggers to begin certain plant development processes for individuals that are photoperiod sensitive (Greenup et al. 2009). Removing the environmental signal, after the metabolic response has been triggered, will not stop the reaction. However, stress may signal the same developmental response as an environmental trigger, but when the stress is removed the reaction halts (Apel and Hirt 2004). Biochemically, when abiotic stress conditions are perceived, the equilibrium between antioxidants and reactive oxygen species (ROS) can become unbalanced causing reactive oxygen stress (Apel and Hirt 2004). The increase in ROS then affects cellular processes and developmental physiology resulting in, for example, reduced seed count, seed weight (Weng et al. 2014) or grain quality (Suriyasak et al. 2017). The differentiation between a physiological response and a stress response in fast generation cycling is important to understand because ROS stress can be mitigated whereas physiological signal responses cannot (Gill and Tuteja 2010). To reduce generation time, it would be desirable to induce a photoperiod response without inducing a stress response.

Another important variable that breeders can manipulate to increase efficiency is the allocation of growing space. Screening larger populations allows for increased selection intensity and therefore results in improved genetic gain. Available glasshouse and field space limits the number of lines that can be advanced per generation. Stress induced by higher planting density could also affect generation time and grain production (Sangoi 2001). Therefore, an investigation into ideal container size in the context of fast generation cycling is warranted.

We investigated fast generation cycling as described by Watson et al. (2018) to determine if generation time in oat could be reduced without embryo rescue. Because oat is sensitive to photoperiod and temperature, we investigated the effect of fast generation cycling and container size on seed production traits, which are important for generation advancement. In addition, we used ascorbic acid measurements to determine if the growth responses were due to induced oxidative stress or photoperiod signaling. 


\section{Materials and Methods}

\section{Plants and growth conditions}

To evaluate the fast generation cycling in oat, we replicated the growth conditions described by Watson et al. (2018) and compared them to glasshouse conditions currently used in the University of Minnesota breeding program.

Four different types of small grains, wheat, 2-row barley, 6-row barley, and oat with four varieties of each (Table $\mathrm{S} 1 *$ ) were grown. Wheat and barley has been previously assessed by Watson et al. (2018) and were therefore used as internal checks. The varieties selected are relevant to the cereal breeding programs at the University of Minnesota. Cereals were grown under both glasshouse and the fast generation conditions described by Watson et al. (2018). The glasshouse temperature ranged between $18{ }^{\circ} \mathrm{C}$ to $30{ }^{\circ} \mathrm{C}$ with light at $500 \mu \mathrm{mol} \mathrm{m} \mathrm{m}^{-2} \mathrm{~s}^{-1}$ during a $14 \mathrm{~h}$ photoperiod.

The growth chamber used for fast generation was set for $27^{\circ} \mathrm{C}$ with light at $500 \mu \mathrm{mol}$ $\mathrm{m}^{-2} \mathrm{~s}^{-1}$ during a $22 \mathrm{~h}$ photoperiod. The chamber was set for four hours at $17^{\circ} \mathrm{C}$ to correspond with the dark $2 \mathrm{~h}$ period similar to the methods described by Watson et al. (2018). Both environments were fertilized the same with a weekly drench of Peters 20-20-20 (ICL, Dublin, OH), Peters' Professional STEM (ICL, Dublin, OH) and a weekly foliar application of calcium nitrate prescribed by Watson et al. (2018).

Container size was evaluated by growing varieties in various volume containers within each environment. The conical shaped containers (i.e. cones) tested were '98-long', '98-short', '200-long', and '200-short' (Stuewe \& Sons Inc., Tangent, OR) with the following soil volumes: $164 \mathrm{ml}, 107 \mathrm{ml}, 66 \mathrm{ml}$, and $49 \mathrm{ml}$, respectively. Varieties were randomized within each sized cone rack for a total of 6 replicates. Rack positions were rotated weekly in both the glasshouse and growth chamber to minimize spatial variation.

Heading date was recorded as the date when the spike or panicle emerged half way or more from the leaf sheath. At grain maturity, the number of fertile tillers was counted and the bulk inflorescences were weighed. Seed from the main inflorescence was hand threshed and counted.

\section{Analysis of ascorbic acid}

For ascorbic acid (AsA) analysis, tissue was collected at Feekes stage 1 and 11 (Large 1954) from the youngest fully mature leaf, to determine how much non-enzymatic reactive oxygen stress each line was exhibiting. Tissue was placed into liquid N2 after sampling to stop metabolism and then stored at $-80{ }^{\circ} \mathrm{C}$ until assayed.

Ascorbic acid (AsA) was measure to determine if the cereals had a non-enzymatic stress response to either environmental conditions or changes in container size. All extraction chemicals were analytical grade and purchased from Sigma-Aldrich (Saint Louis, MO). Estimation of AsA was conducted with $100 \pm 10 \mathrm{mg}$ of plant tissue. The tissue was homogenized with sterile distilled water for 10 minutes then centrifuged $(14,034 \mathrm{~g})$ for

\footnotetext{
*Further details about the Electronic Supplementary Material (ESM) can be found at the end of the article.
} 
seven minutes at $4{ }^{\circ} \mathrm{C}$. AsA was extracted from the supernatant using an adapted method from (Nath et al. 2014) with a mixture containing sodium molybdate $(2 \% \mathrm{w} / \mathrm{v}), 0.5 \mathrm{M}$ sulfuric acid $\left(\mathrm{H}_{2} \mathrm{SO}_{4}\right)$, and $0.5 \mathrm{M}$ sodium phosphate $\left(\mathrm{Na}_{3} \mathrm{PO}_{4}\right)$. The mixture was incubated at $60{ }^{\circ} \mathrm{C}$ for 40 minutes and centrifuged $(14,034 \mathrm{~g})$ for 1 minute. The resulting supernatant absorbance was recorded at $660 \mathrm{~nm}$ with a microplate spectrophotometer (Biotek, Winooski, VT) and final AsA concentrations were calculated using a standard curve.

\section{Statistics}

A two-way analysis of variance (ANOVA) and mean comparison using Tukey HSD was performed on experiments within cereals and between experimental conditions (i.e. growth conditions and container size) using JMP 3.3.0 (SAS Institute Inc., Cary, NC). Varieties were considered a random effect. All results are a mean of 6 replicates.

\section{Results}

\section{Fast generation cycling}

To determine the impact of fast generation cycling on oat, cereals grown in a glasshouse under normal light conditions were compared to plants grown under an extended photoperiod within a growth chamber. For comparison between the two environments, only large cones $(164 \mathrm{ml})$ were assessed. All cereals reached anthesis faster when placed in the fast generation cycling environment $(P<0.0001)$ (Fig. 1a). The fast generation conditions decreased days to anthesis by $15 \pm 3$ days averaged across all cereals.

Fast generation cycling environment did impact seed count for oat and 6-row barley $(P<0.0001)$ by $67 \%$ and $30 \%$ respectively (Fig. 1 b). The wheat and 2 -row barley were not affected. Interestingly, 6-row barley total inflorescence weight at maturity did not change with the reduction in seed count (Fig. 1c), whereas oat showed a corresponding reduction in inflorescence weight $(P<0.0001)$ with the decrease of seeds per inflorescence.

Each cereal had a different tillering response to the change in environment (Fig. 1d). Barley, both 2-row and 6-row, decreased in tiller production in response to fast generation cycling $(P \leq 0.03$ and $P<0.0001$, respectively), while oat and wheat had no discernable difference in tiller count between the two environments $(P \geq 0.10$ and $P \geq 0.60$, respectively).

The compound AsA was measure to determine if fast generation cycling induced a non-enzymatic stress response. All cereals increased in AsA concentration as the plants reached maturity by approximately 4 fold compared to the seedling stage. Oat seedlings appeared to increase production of AsA in response to fast generation conditions compared to glasshouse controls $(P \geq 0.08)$ (Table 1$)$, but not to the extent of barley $(P \leq 0.01)$, (Table S2 and S3). As a plant matured, the differences in AsA concentrations diminished between the fast generation and glass house environments, suggesting that all cereals eventually recovered from seedling ROS (Fig. S1). 
A

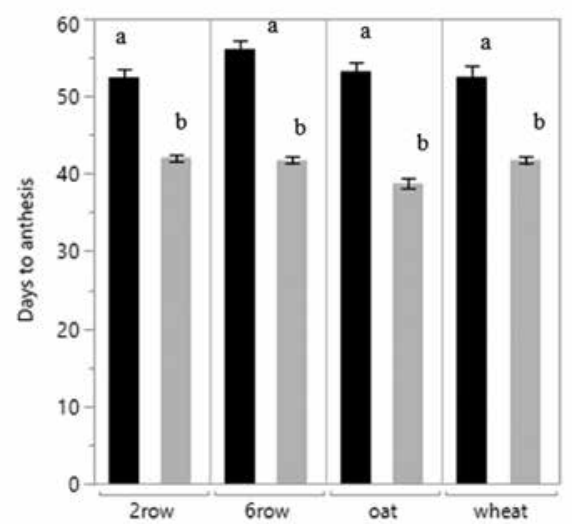

C

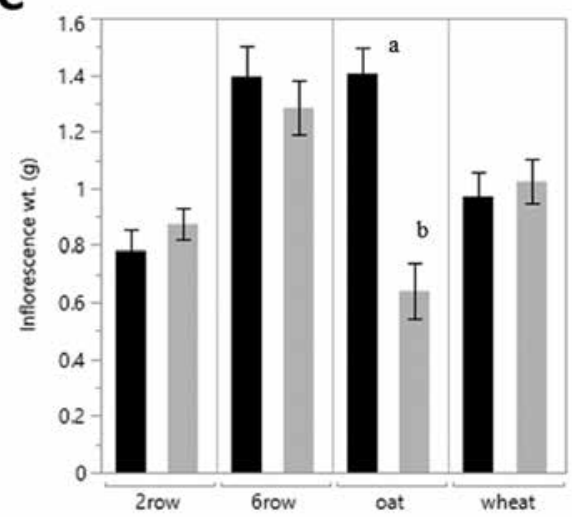

B

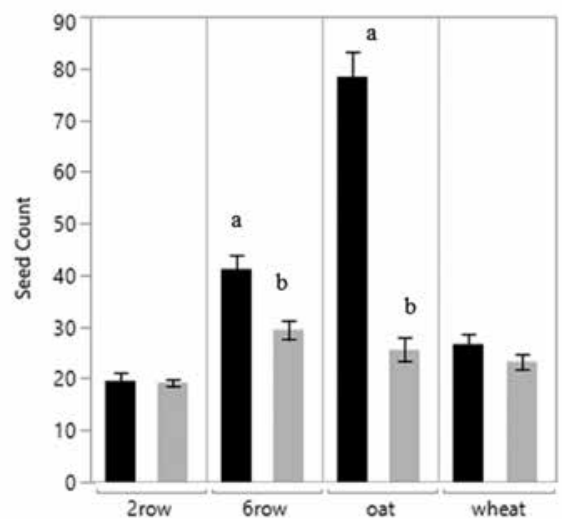

D

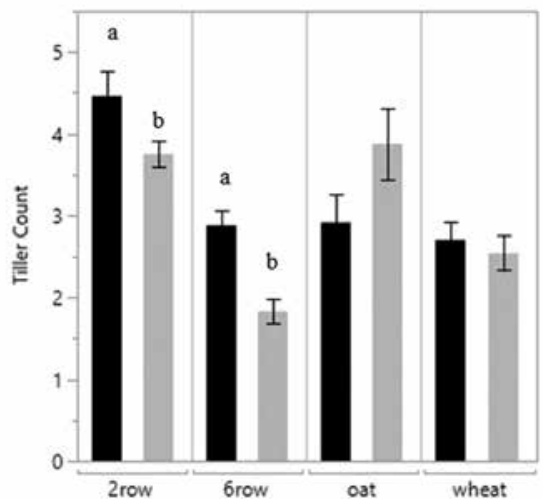

Figure 1. The impact of fast generation cycling conditions compared to glasshouse condition for $\mathrm{A}-$ days to anthesis; B - seed count; C - individual inflorescence weight (g); and D - tiller number. Black bars indicate glasshouse and grey indicate fast generation cycling conditions. Letters indicate significance within cereals using Tukey HSD with $\alpha<0.05$

\section{Container size}

We planted cereals in four different sized containers and grew the plants under both environmental conditions to determine the ideal container size. Container size had no significant difference on time to anthesis for oat when grown under either growth condition (Table 1). The overall seed number, inflorescence weight and tiller count for oat was significantly impacted by container size. When identical cone sizes were compared in different growth conditions, agronomic traits, with the exception of tiller count, had a reduction in value in the fast generation cycle conditions compared to the glass house 


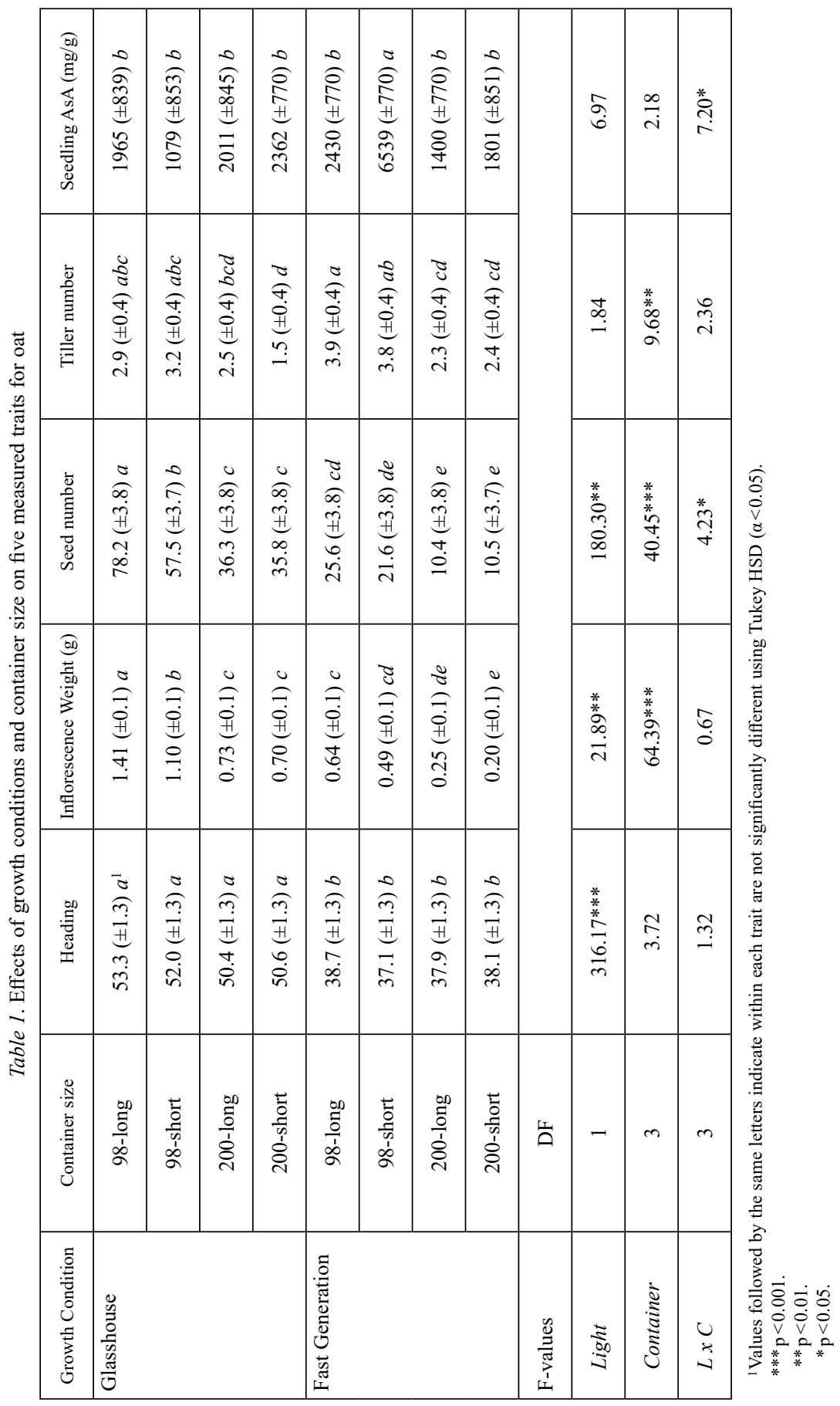

Cereal Research Communications 47, 2019 
(Table 1). Normal glass house conditions and large cones resulted in more seed and higher inflorescence weight, whereas, fast generation cycling conditions and small cones resulted in low seed count and inflorescence weight for all cereals (Tables S2-S4). Tiller count for oat and the other cereals collectively were strongly linked to cone size irrespective of the growth environment $(P \leq 0.001)$ with the exception of 2-row barley $(P \leq 0.05)$ (Table S3).

AsA, measured to determine the effects of ROS stress on container size, in oat did not significantly change between container sizes or growth conditions. However, there is an interaction between growth conditions and container sizes for oat (Table 1).

\section{Discussion}

The purpose of this study was to evaluate the potential of using fast generation techniques in an oat breeding program to reduce generation time. Any reduction of generation time has the potential to benefit a breeding program, either by increasing the numbers of potential generations that can be moved forward per year or by accommodating unexpected delays within the breeding pipeline. We were able to verify that fast generation cycling conditions reduced the time to anthesis and grain maturity for oat, with a few considerations.

In oat, reduction of days to anthesis are similar to what was reported in the Queensland study for wheat and barley (Watson et al. 2018), suggesting that is method is viable for fast generation cycling in oat without the need for embryo rescue. The fast cycling growth conditions reduced seed count per oat plant and the corresponding seed weight per inflorescence (Fig. 1). Oats are known for being sensitive to high light intensity by inhibiting photosynthesis (Quiles 2006). The impairment of photosynthesis may retard cell division. If cell division halts during flower primordia development, then a reduction of eventual seeds produced could occur (Slafer et al. 2002). If a breeder is utilizing single seed decent to advance generations, this fast generation cycling conditions should be effective since only one (or a few) seeds are required.

Oat was the only cereal where AsA concentration did not increase (Table 1) in response to fast generation cycling conditions. Insufficient concentrations of AsA to control ROS would result in the disruption of normal plant metabolic functions that could affect seed production (Gill and Tuteja 2010). Our results suggest that the reduction in seed count and inflorescence weight is a stress response since plants at equilibrium between antioxidants (i.e. AsA) and ROS, are able to maintain plant development (Apel and Hirt 2004). This stress on oat may impact seedling vigor of future generations (Grass and Burris 1995) and therefore influence how the resulting fast generation grown seed can be use within a breeding program. For example, seed produced from stressed maternal plants may have reduced vigor that could affect seedling phenotyping.

Another benefit of using fast generation cycling could be the ability to synchronize flowering time between early and late flowering genotypes to facilitate cross pollination. Reducing the overall time of flowering (Araus et al. 2002; Slafer et al. 2002; Miralles et al. 2000), could reduce the variation for anthesis among genotypes. The reduction of 
inflorescence initiation time, which coincides with the beginning of stem elongation (Miralles et al. 2000; Webster and Wood 2011), may have led to the two-thirds reduction of seed count observed in oat (Fig. 1). Therefore, the trade-off between the reduction in generation time and seed count may be problematic depending on breeding goals.

Wheat and 2-row barley appear best suited for this method of fast generation cycling because there was no change in yield components (i.e. seed count or seed weight) compared to glasshouse controls (Fig. 1) associated with the shortening of days to anthesis. However, earlier studies of wheat indicate a reduction of seed count (Fisher 1985) and seed weight (Araus et al. 2002) corresponding with increased photoperiod, suggesting that light is not independently affecting yield components. Interestedly, even though 2-row and 6-row barley are the same species, each type responded differently to environmental conditions (Fig. 1), suggesting physiological diversity between the two barley types.

We found that the reduction in container space, irrespective of environmental conditions, affected agronomic traits for all cereals. For seed traits (count and inflorescence weight), the response was more dramatic under the extended photoperiod (Table 1) than glasshouse controls. Plants grown in containers commonly are exposed to nutrient and drought stress due to the limited space for root expansion which is a key method for drought avoidance (Verslues et al. 2006). Even with careful attention to watering within a glasshouse, mild water stress can occur. Avoidance and tolerance mechanisms, such as reactive oxygen scavenging, can be sufficient to maintain plant performance (i.e. seed production) (Kramer and Boyer 1995). Therefore, the use of fast generation method proposed by Watson et al. (2018) can be successfully applied to oat, and is less labor intensive than current oat fast generation cycling methods. However, breeding goals must be considered when using this fast generation technique; for oat, this method would be ideal for moving populations ahead using single seed descent, but it is not practical for situations where a large seed count or strong seedling vigor are required to evaluate traits on replicated plants (e.g. disease reaction).

\section{Acknowledgements}

We thank the lab contributions by Dimitri Von Ruckert and Joan Barretto. This project was supported in part with grant funds from Pepsi Co.

\section{References}

Apel, K., Hirt, H. 2004. Reactive oxygen species: metabolism, oxidative stress, and signal transduction. Annu. Rew. Plant Biol. 55:373-399.

Araus, J.L., Slafer, G.A., Reynolds, M.P., Royo, C. 2002. Plant breeding and drought in C3 cereals: what should we breed for? Ann. Bot.-London 89(7):925-940.

Chowdhury, S.I., Wardlaw, I.F. 1978. The effect of temperature on kernel development in cereals. Aust. J. Agr. Res. 29:205-223.

Gill, S.S., Tuteja, N. 2010. Reactive oxygen species and antioxidant machinery in abiotic stress tolerance in crop plants. Plant Physiol. Bioch. 48(12):909-993. 
Grass, L., Burris, J.S., 1995. Effect of heat stress during seed development and maturation on wheat (Triticum durum) seed quality. I. Seed germination and seedling vigor. Can. J. Plant. Sci. 75(4):821-829.

Greenup, A., Peacock, W.J., Dennis, E.S., Trevaskis, B. 2009. The molecular biology of seasonal floweringresponses in Arabidopsis and the cereals. Ann. Bot.-London 103(8):1165-1172.

Fischer, R. 1985. Number of kernels in wheat crops and the influence of solar radiation and temperature. J. Agr. Sci. 105(2):447-461.

Forster, B.P., Till, B.J., Ghanim, A.M.A., Huynh, H.O.A., Burstmayr, H., Caligari, P.D.S. 2014. Accelerated plant breeding. CAB Review 9:1-16.

Kramer, P.J., Boyer, J.S. 1995. Water relations of plants and soils. Academic Press. New York, USA

Large, E.C. 1954. Growth stages in cereals, illustration of the feekes scale. Plant Pathol. 3:128-129.

Liu, H., Zwer, P., Wang, H., Liu, C., Lu, Z., Wang, Y., Yan, G. 2016. A fast generation cycling system for oat and triticale breeding. Plant Breeding 135(5):574-579.

Miralles, D.J., Richards, R.A., Slafer, G.A. 2000. Duration of the stem elongation period influences the number of fertile florets in wheat and barley. Funct. Plant Biol. 27(10):931-940.

Nath, S., Panda, P., Mishra, S., Dey, M., Choudhury, S., Sahoo, L., Panda, S.K. 2014. Arsenic stress in rice: Redox consequences and regulation by iron. Plant Physiol. Bioch. 80:203-210.

Quiles, M.J. 2006. Stimulation of chlororespiration by heat and high light intensity in oat plants. Plant Cell Environ. 29(8): $1463-1470$.

Rines, H.W. 2003. Oat haploids from wide hybridization. In: Małuszyński, M. et al. (eds), Double Haploid Production in Crop Plants. Kluwer Academic Publishers. Dordrecht, The Netherlands, pp. 155-159.

Sangoi, L. 2001. Understanding plant density effects on maize growth and development: an important issue to maximize grain yield. Ciência rural. 31(1):159-168.

Slafer, G.A., Molina-Cano, J.L., Savin, R., Araus, J.L., Romagosa, I., 2002. Barley science: Recent advances from molecular biology to agronomy of yield and quality. CRC Press.

Stockman, Y.M., Fischer, R.A., Brittain, E.G. 1983. Assimilate supply and floret development within the spike of wheat (Triticum aestivum L.). Funct. Plant Biol. 10:585-594.

Suriyasak, C., Harano, K., Tanamachi, K., Matsuo, K., Tamada, A., Iwaya-Inoue, M., Ishibashi, Y. 2017. Reactive oxygen species induced by heat stress during grain filling of rice (Oryza sativa L.) are involved in occurrence of grain chalkiness. J. Plant Physiol. 216:52-57.

Verslues, P.E., Agarwal, M., Katiyar-Agarwal, S., Zhu, J., Zhu, J.K. 2006. Methods and concepts in quantifying resistance to drought, salt and freezing, abiotic stresses that affect plant water status. Plant. J. 45(4):523539.

Watson, A., Ghosh, S., Williams, M.J., Cuddy, W.S., Simmonds, J., Rey, M.-D., Hatta, M.A., Hinchliffe, A., Steed, A., Reynolds, D., Adamski, N.M., Breakspear, A., Korolev, A., Rayner, T., Dixon, L.E., Riaz, A., Martin, W., Ryan, M., Edwards, D., Batley, J., Raman, H., Carter, J., Rogers, C., Domoney, C., Moore, G., Harwood, W., Nicholson, P., Dieters, M.J., DeLacy, I.H., Zhou, J., Uauy, C., Boden, S.A., Park, R.F., Wulff B.B.H., Hickey, L.T. 2018. Speed breeding is a powerful tool to accelerate crop research and breeding. Nat. Plants 4:23-29.

Webster, F.H., Wood, P.J. 2011. Oats Chemistry and Technology. $2^{\text {nd }}$ ed. St. Paul: AACC International, Inc.

Weng, X., Wang, L., Wang, J., Hu, Y., Du, H., Xu, C., Xing, Y., Li, X., Xiao, J., Zhang, Q. 2014. Grain number, plant height, and heading date is a central regulator of growth, development, and stress response. Plant Physiol. 164(2):735-747.

\section{Electronic Supplementary Material (ESM)}

Electronic Supplementary Material (ESM) associated with this article can be found at the website of CRC at http://www.akademiai.com/content/120427/

Electronic Supplementary Table S1. List of small grains genotypes grown 
Electronic Supplementary Table S2. Effects of growth conditions and container size on five measured traits for wheat

Electronic Supplementary Table S3. Effects of growth conditions and container size on five measured traits for 2-row barley

Electronic Supplementary Table S4. Effects of growth conditions and container size on five measured traits for 6-row barley 